\title{
Projeto "criança saudável, educação dez": resultados com e sem intervenção do nutricionista ${ }^{1}$
}

\author{
"Healthy child education project, the best \\ education": results with and without \\ the dietician's intervention
}

Cláudia Rucco Penteado DETREGIACHI ${ }^{2}$

Tânia Moron Saes BRAGA ${ }^{3}$

RE S U M O

\section{Objetivo}

Planejar e aplicar um programa de orientação em nutrição, realizado por nutricionista e destinado a professores de $1^{a}$ a $4^{a}$ série do ensino fundamental, visando analisar a contribuição desse profissional no resultado do projeto "Criança Saudável, Educação Dez" quanto ao conhecimento dos escolares.

\section{Métodos}

O trabalho foi realizado em duas escolas públicas, A e B, nas quais foi desenvolvido o projeto educativo, sendo que na primeira os professores foram apoiados por um programa de orientação realizado por profissional nutricionista. Antes e depois do desenvolvimento do projeto educativo foi aplicado um instrumento para avaliar o conhecimento dos escolares sobre alimentação e nutrição.

\section{Resultados}

Os escolares da Escola A apresentaram mudança significante $(p<0,01)$ no conhecimento de alimentação e nutrição após o desenvolvimento do projeto educativo, o que não ocorreu entre os estudantes da Escola B (p>0,05).

\section{Conclusão}

Os resultados obtidos sugerem que a realização de programa de orientação, prestada por nutricionistas aos professores para desenvolverem o projeto educativo, maximizou os resultados, gerando mudança significante

1 Artigo elaborado a partir da tese de C.R.P. DETREGIACHI, intitulada "Programa de orientação a professores para o desenvolvimento de projeto educativo: efeito na aprendizagem acerca de alimentação saudável". Universidade Estadual Paulista Júlio de Mesquita Filho; 2008.

2 Universidade Estadual Paulista Júlio de Mesquita Filho, Instituto de Biociências de Botucatu, Departamento de Educação. Campus Botucatu, Caixa Postal 510, Distrito de Rubião Júnior, s/n., 18618-970, Botucatu, SP, Brasil. Correspondência para/ Correspondence to: C.R.P. DETREGIACHI. E-mails: <claudiarucco@flash.tv.br>; <claudiarucco@ibb.unesp.br>.

${ }^{3}$ Universidade Estadual Paulista Júlio de Mesquita Filho, Faculdade de Filosofia e Ciências, Departamento de Educação Especial. Marília, SP, Brasil. 
no conhecimento dos escolares sobre alimentação e nutrição. Tais resultados apontam a importância da capacitação do nutricionista para atuar em educação nutricional, indicando que o campo pedagógico merece aprofundamento nos cursos de graduação, de modo a permitir ao aluno e futuro profissional uma compreensão alicerçada e sólida do processo educativo.

Termos de indexação: Crianças. Educação alimentar e nutricional. Ensino superior. Nutricionista.

\section{A B S T R A C T}

\section{Objective}

In this study, a dietician planned and implemented a nutrition counseling program for first through fourth grade elementary school teachers. The objective was to analyze the contribution of this professional to the result of the project "Healthy Child, Outstanding Education", regarding the students' knowledge.

\section{Methods}

The program was implemented in two public schools - A and B, in which an education project was developed. In School A, the teachers had the support of a counseling program provided by a dietician. The students' knowledge on food and nutrition was assessed before and after the implementation of the education project.

\section{Results}

Knowledge on food and nutrition among School A students changed significantly $(p<0.01)$ after the implementation of the education program. This did not occur among School B students $(p>0.05)$.

\section{Conclusion}

The results suggest that the counseling program administered by a dietician for teachers to develop the education project maximized the results. The knowledge gained by the students on food and nutrition was significant. These results show the importance of training a dietician to provide nutrition education. The education field deserves more attention in undergraduate courses so that the student and future professional gain a solid and well-founded understanding of the education process.

Indexing terms: Children. Feed and nutritional education. Education, higher. Nutritionist.

\section{N T R O D U Ç Ã O}

Com a finalidade de concretizar o direito humano à alimentação e à nutrição adequadas, em 1999 foi homologada a Política Nacional de Alimentação e Nutrição (PNAN) ${ }^{1}$, cujas diretrizes foram incorporadas à atual Política Nacional de Saúde. A PNAN tem como objetivo principal contribuir para o conjunto de políticas governamentais voltadas à concretização daquele direito, bem como à garantia da segurança alimentar e nutricional da população. Em vista disso, o Brasil busca implementar as recomendações preconizadas pela Organização Mundial da Saúde no âmbito da Estratégia Global de Promoção da Alimentação Saudável, Atividade Física e Saúde².

A PNAN tem como diretrizes a promoção de práticas alimentares saudáveis, a prevenção e controle dos distúrbios nutricionais e das doenças associadas à alimentação e nutrição, o monitoramento da situação alimentar e nutricional, a garantia da qualidade dos alimentos colocados para consumo no país, o desenvolvimento de pesquisas e recursos humanos, bem como o estímulo às ações intersetoriais que propiciem o acesso universal aos alimentos ${ }^{1}$. Tais diretrizes, embora planejadas para serem implementadas no âmbito do setor de saúde, extrapolam para outros setores governamentais e não governamentais, por meio de um conjunto de ações realizadas em parceria.

A promoção de práticas alimentares saudáveis, além de ser uma diretriz explícita da PNAN, conforma uma ação transversal incorporada em todas as outras ações, programas e projetos. Nessa abordagem, dá-se enfoque prioritário a hábitos e práticas alimentares regionais, resgatando o consumo de alimentos locais de 
elevado valor nutritivo, bem como incentivando padrões alimentares mais variados desde os primeiros anos de vida até a idade adulta e velhice ${ }^{1}$.

Com base nas recomendações da Estratégia Global para Alimentação Saudável, Atividade Física e Saúde ${ }^{2}$, nas diretrizes da PNAN ${ }^{1}$ e na premissa de que os hábitos alimentares adquiridos na infância tendem a se solidificar na vida adulta e afetam o comportamento alimentar do indivíduo por toda a vida 3,4 , em 2006 foram instituídas as diretrizes para a Promoção da Alimentação Saudável nas Escolas, englobando as instituições públicas e privadas de educação infantil, fundamental e nível médio, em âmbito nacional ${ }^{5}$. Essas diretrizes preveem, entre outras ações, a incorporação do tema "alimentação saudável" no projeto político pedagógico da escola, contemplando o desenvolvimento contínuo de programas de promoção de hábitos alimentares saudáveis.

Nesse sentido, com vista à consolidação da promoção da alimentação saudável no ambiente escolar, foi implementado o projeto "Criança Saudável, Educação Dez", uma ação do Programa Fome Zero. O Ministério do Desenvolvimento Social e Combate à Fome (MDS) contou com o apoio dos Ministérios da Saúde e Educação, assim como do Fundo Nacional de Desenvolvimento da Educação (FNDE)6. Firmou ainda um contrato com a Editora Globo, produzindo material educativo composto por revistas em quadrinhos, com personagens de Monteiro Lobato, que abordam os temas educação nutricional, meio ambiente e cidadania. O material foi distribuído para escolares da $1^{\text {a }}$ à $4^{\text {a }}$ série do ensino fundamental das escolas públicas de todo o país, nos anos de 2005 e 2006. Para os docentes responsáveis por essas turmas, foi enviado o caderno do professor a fim de apoiar e direcionar o trabalho em sala de aula.

A importância da educação nutricional justifica a atuação governamental no sentido de desenvolver e promover programas que favoreçam escolhas alimentares saudáveis, em nível individual e coletivo. Ademais, Boog ${ }^{7}$ aponta a necessidade de se criarem espaços institucionais que garantam o desenvolvimento de ações de educação nutricional, abrangendo planejamento, implementação e avaliação dessas ações, assim como investimento no aprimoramento dos profissionais da área.

Nesse sentido, para o desenvolvimento de programas efetivos de educação nutricional no ambiente escolar, Gaglianone ${ }^{8}$ recomenda que os professores sejam adequadamente treinados. Adicionalmente, a Portaria Interministerial $1.010^{5}$ determina que, para a promoção da alimentação saudável nas escolas, deve-se incluir a capacitação de profissionais de saúde e de educação, entre outros. Nesse contexto, a American Dietetic Association ${ }^{9}$ assim como Bizzo \& Leder ${ }^{10}$ destacam ser importante que o profissional especialista em nutrição participe dos programas de educação nutricional, o que é essencial para a efetividade de tais programas e para a aquisição de mudanças desejáveis no estado nutricional da população.

Assim, vê-se a importância do componente educação nutricional na formação do nutricionista. A abordagem educacional está prevista nas Diretrizes Curriculares do Curso de Graduação em Nutrição"11 por meio de disciplinas nas áreas de ciências sociais e humanas. De outro lado, Canesqui \& Garcia ${ }^{12}$ observaram que apenas uma pequena carga horária da matriz curricular é destinada para essas áreas.

Dado o desafio imposto aos nutricionistas em prol da promoção da alimentação saudável no ambiente escolar, o presente estudo teve como objetivo o planejamento e a aplicação de um programa de orientação em nutrição. O programa foi realizado por nutricionista e destinado a professores de $1^{\mathrm{a}}$ a $4^{\mathrm{a}}$ série do ensino fundamental de escolas públicas, visando à análise da contribuição daquele profissional nos resultados do projeto "Criança Saudável, Educação Dez" quanto ao conhecimento dos escolares.

\section{MÉTODOS}

O estudo caracteriza-se por uma investigação realizada com escolares da $1^{\mathrm{a}}$ à $4^{\mathrm{a}}$ série do 
ensino fundamental de duas escolas públicas, uma municipal (Escola A) e outra estadual (Escola B), da cidade de Marília (SP), nas quais foi desenvolvido o projeto educativo "Criança Saudável, Educação Dez". O trabalho trata da avaliação do conhecimento dos escolares sobre alimentação e nutrição, realizada antes (Avaliação inicial) e depois (Avaliação final) do desenvolvimento do programa de educação nutricional.

O desenvolvimento do projeto educativo na Escola A contou com um programa de orientação aos professores da $1^{a}$ à $4^{a}$ série do ensino fundamental e seus substitutos, realizado por uma nutricionista, com o intuito de apoiá-los nas possíveis lacunas do conhecimento sobre o assunto, visto que são profissionais da educação não necessariamente estudiosos do tema. Já na Escola $B$, o projeto educativo foi desenvolvido sem a existência dessas condições, tal como estava previsto no planejamento do MDS.

A opção por utilizar uma escola municipal e uma estadual justifica-se pelo fato de serem as únicas escolas públicas que não haviam utilizado o material educativo do projeto "Criança Saudável, Educação Dez" até o momento da realização dos procedimentos preliminares.

A Escola A possuía 18 salas de $1^{\text {a }}$ a $4^{\mathrm{a}}$ série do ensino fundamental, distribuídas entre os turnos da manhã e tarde, totalizando 499 escolares matriculados, enquanto a Escola B contava com 239 alunos matriculados naquelas séries e distribuídos em 8 classes. Dentre os alunos da Escola A, participaram 313 escolares, representando $63 \%$ da população, com idade média de 8,8 anos (Desvio-Padrão - DP=1,7 ano). Na Escola B optou-se por utilizar uma amostra de conveniência, parcial e aleatória, do total de escolares matriculados, sendo avaliada uma classe de cada série, compondo uma amostra de 126 alunos, correspondente a 53\% dos matriculados. Dos 126 escolares que compunham a amostra do estudo nessa escola, 76 participaram da avaliação, representando $60 \%$ da amostra, com média de idade de 8,9 anos ( $D P=1,8$ ano).
Na Escola A, participaram do programa de orientação os 18 professores responsáveis pelas classes de $1^{\text {a }}$ a $4^{\text {a }}$ série, além de 3 professores substitutos, totalizando 21 docentes participantes do estudo.

O programa de orientação aos professores da Escola A ocorreu durante o Horário de Estudo Coletivo (HEC), simultaneamente ao desenvolvimento do projeto educativo "Criança Saudável, Educação Dez", utilizando como base o caderno do professor que compunha o material do projeto. O programa foi composto por oito encontros com duração aproximada de 60 minutos cada um, totalizando 8 horas de estudos. Os encontros contaram com a participação dos professores e seus substitutos, bem como da coordenadora pedagógica da escola. Foi adotada a abordagem dialógica para o aprofundamento teórico-reflexivo do conteúdo do projeto educativo, com a valorização das falas dos professores, marcadas por sua realidade pedagógica em sala de aula, buscando assim romper o modelo de transmissão e recepção de informação. A posição da pesquisadora foi de condução não diretiva das discussões, permitindo a troca de experiências e de saberes entre os participantes na busca de ampliação e fortalecimento dos conceitos sobre alimentação e nutrição, e tendo como princípio que os conteúdos seriam abordados com base na realidade dos alunos sem se afastar da proposta do projeto educativo. Nessa oportunidade também era programada a confecção do material de apoio e/ou procedimentos necessários para o desenvolvimento das atividades. O modelo de ação educativa delineado buscou proporcionar aos professores estímulo ao desenvolvimento de habilidades a serem empregadas no seu cotidiano profissional junto aos escolares, ampliando sua autonomia e incentivando a atitude protagonista no campo da educação nutricional.

Para a coleta de dados foi utilizado um instrumento elaborado pela pesquisadora, com o objetivo de avaliar o conhecimento dos escolares das Escolas A e B sobre alimentação e nutrição. A versão inicial do instrumento foi respondida como 
pré-teste pelos alunos de $1^{\mathrm{a}}$ a $4^{\mathrm{a}}$ série de uma escola municipal de ensino fundamental, a fim de verificar a compreensão das questões e a melhor forma de apresentação aos escolares no momento da aplicação. Após a realização do pré-teste, observou-se que as questões apresentavam bom nível de compreensão, porém ainda exigiam algumas adequações nos seus enunciados. Percebeu-se também a necessidade da leitura do enunciado antes da realização de cada questão, a fim de possibilitar o correto entendimento da mesma. Com o intuito de padronizar a utilização do instrumento junto aos escolares, um protocolo foi elaborado e seguido pela pesquisadora. A professora responsável pela classe escolar foi esclarecida de que era facultativa sua permanência na sala enquanto os alunos respondessem às questões. Porém, se optasse por permanecer, deveria abster-se de qualquer manifestação de auxílio aos escolares no preenchimento do questionário. O instrumento foi respondido pelos alunos antes do início do desenvolvimento do projeto educativo "Criança Saudável, Educação Dez" (Avaliação inicial), assim como imediatamente após seu término (Avaliação final).

Para padronizar o desenvolvimento do projeto na escola municipal e permitir a organização do programa de orientação aos professores, foi estabelecido que todas as classes desenvolveriam o projeto simultaneamente, segundo um cronograma previamente elaborado pela pesquisadora.

A realização do estudo foi submetida ao Comitê de Ética em Pesquisa da Universidade Estadual Paulista Júlio de Mesquita Filho (UNESP) - campus de Marília, sendo aprovada conforme o Parecer n²208/2005.

\section{RESULTADOS}

Entre os 499 escolares matriculados entre a $1^{\text {a }}$ e a $4^{\mathrm{a}}$ série da Escola A, 313 participaram da avaliação inicial e final, representando 63\% da população. Na Escola B, dos 126 escolares que compunham o grupo estudado, 76 participaram da avaliação, representando 60\% da amostra.

A perda amostral em ambas as escolas foi devida à ausência do escolar em sala de aula no dia da aplicação do instrumento ou à inconsistência no preenchimento do mesmo. Foram considerados na pesquisa somente os dados daqueles escolares que participaram nos dois momentos das avaliações, inicial e final.

Na avaliação do conhecimento sobre alimentação e nutrição, a cada escolar foi atribuído um escore, com possibilidade de variação de 14 pontos negativos a 36 pontos positivos, em que os extremos caracterizam maior ocorrência de erro e de acerto, respectivamente. Posteriormente, foi feita a comparação dos escores obtidos pelos escolares antes (inicial) e depois (final) do desenvolvimento do projeto educativo (Tabelas 1 e 2), por meio da aplicação do teste $t$ para observações pareadas ${ }^{13}$.

Os resultados obtidos com a análise estatística mostram que, no nível de significância de $1 \%$, o projeto educativo gerou mudança $(p<0,01)$ no conhecimento sobre alimentação e nutrição entre os escolares da $1^{a}$ à $4^{a}$ série do ensino fundamental da Escola A. Porém, entre os escolares da Escola B, os resultados obtidos indicam que, no nível de significância de $5 \%$, o projeto educativo não gerou mudança no conhecimento sobre alimentação e nutrição $(p>0,05)$, com exceção apenas para os matriculados na $3^{a}$ série $(p<0,05)$.

Tabela 1. Escores obtidos pelos escolares da Escola A na avaliação do conhecimento sobre alimentação e nutrição, antes e depois do desenvolvimento do projeto educativo. Marília (SP), 2008.

\begin{tabular}{|c|c|c|c|c|c|c|c|c|}
\hline \multirow{4}{*}{ Escola A grupo-intervenção } & \multicolumn{8}{|c|}{ Média dos Escores } \\
\hline & \multicolumn{2}{|c|}{$1^{\text {a }}$ série $n=77$} & \multicolumn{2}{|c|}{$2^{\mathrm{a}}$ série $\mathrm{n}=82$} & \multicolumn{2}{|c|}{$3^{a}$ série $n=55$} & \multicolumn{2}{|c|}{$4^{\text {a }}$ série $n=98$} \\
\hline & Inicial & Final & Inicial & Final & Inicial & Final & Inicial & Final \\
\hline & 12,4 & 22,6 & 16,9 & 24,2 & 20,4 & 26,1 & 22,6 & 27,2 \\
\hline & \multicolumn{2}{|c|}{$<0,01$} & \multicolumn{2}{|c|}{$<0,01$} & \multicolumn{2}{|c|}{$<0,01$} & \multicolumn{2}{|c|}{$<0,01$} \\
\hline
\end{tabular}


56 | C.R.P. DETREGIACHI \& T.M.S. BRAGA

Tabela 2. Escores obtidos pelos escolares da Escola B na avaliação do conhecimento sobre alimentação e nutrição, antes e depois do desenvolvimento do projeto educativo. Marília (SP), 2008.

\begin{tabular}{|c|c|c|c|c|c|c|c|c|}
\hline \multirow{4}{*}{ Escola B grupo-controle } & \multicolumn{8}{|c|}{ Média dos Escores } \\
\hline & \multicolumn{2}{|c|}{$1^{\text {a }}$ série $n=23$} & \multicolumn{2}{|c|}{$2^{a}$ série $n=15$} & \multicolumn{2}{|c|}{$3^{a}$ série $n=15$} & \multicolumn{2}{|c|}{$4^{a}$ série $n=3$} \\
\hline & Inicial & Final & Inicial & Final & Inicial & Final & Inicial & Final \\
\hline & 18 & 16,9 & 14,2 & 15,6 & 24,2 & 26,4 & 28,4 & 25,2 \\
\hline & \multicolumn{2}{|c|}{$<0,05$} & \multicolumn{2}{|c|}{$<0,05$} & \multicolumn{2}{|c|}{$<0,05$} & \multicolumn{2}{|c|}{$<0,05$} \\
\hline
\end{tabular}

O efeito do projeto educativo sobre o conhecimento deu-se de forma diferente entre os escolares das Escolas A e B. Entre os escolares da Escola B, as mudanças no conhecimento sobre alimentação e nutrição não ocorreram de forma significante, apresentando $p>0,05$ em três das quatro séries escolares avaliadas (Tabela 2), acrescido do fato de os escolares da $1^{\mathrm{a}}$ e $4^{\mathrm{a}}$ séries, após desenvolvimento do projeto educativo, apresentarem escore menor do que aquele obtido na avaliação inicial.

\section{DIS CUSS Ã O}

A distinção nos resultados obtidos pelos escolares das Escolas A e B no conhecimento de alimentação e nutrição mediante o desenvolvimento do projeto de educação nutricional pode ser atribuída à realização do programa de orientação por nutricionista, como forma direta de apoio aos professores da Escola A. Infere-se que o programa proporcionou a esses docentes um aprofundamento teórico-reflexivo em relação a possíveis lacunas de conhecimento sobre nutrição, fato que não ocorreu na Escola B, embora tivesse utilizado o mesmo material de apoio no desenvolvimento do projeto.

Nesse sentido, Davanço et al. ${ }^{14}$ observaram que professores expostos a programa de treinamento para o desenvolvimento de projeto educativo apresentaram melhor preparo em termos de conteúdo, tendo esse resultado sido significativamente diferente em relação a professores que não passaram por treinamento. Os autores também observaram que, comparado ao grupo-controle, o grupo de professores treinado apre- sentou atitudes mais positivas em relação ao desenvolvimento de programas de educação nutricional na escola, o que pôde gerar maior envolvimento e responsabilidade com a promoção de ações de saúde junto aos escolares e, consequentemente, melhores resultados.

Cabe ainda ressaltar o fato de os escolares da $1^{\text {a }}$ e $4^{\mathrm{a}}$ séries da Escola B terem apresentado na avaliação final escore menor do que aquele obtido na avaliação inicial. Tal resultado suscita a reflexão sobre o efetivo desenvolvimento do projeto educativo em meio às demandas do ensino regular, bem como sobre a conscientização dos professores acerca de seu papel na educação nutricional. Observa-se que na Escola B não houve o desenvolvimento do programa de orientação aos professores, impossibilitando a sensibilização dos educadores e a discussão de situações de aprendizagem semelhantes àquelas que seriam vivenciadas em sala de aula, condições que podem ter limitado a construção do conhecimento.

Embora este estudo não tenha medido o conhecimento dos professores antes e depois do desenvolvimento do programa de orientação, pode-se pensar que os professores da Escola $A$ provavelmente adquiriram maior conhecimento e melhores habilidades em trabalhar adequadamente com o tema em questão, visto os melhores resultados alcançados por seus alunos. Adicionalmente, a realização de tal programa gerou entre os participantes a discussão ampla sobre o tema alimentação e nutrição, não se restringindo apenas ao conteúdo proposto no projeto educativo, além de ter proporcionado o envolvimento de toda a equipe pedagógica, inclusive com a participação da coordenadora e interveniências positivas da diretora da escola. 
Macedo ${ }^{15}$, analisando estratégia de capacitação em educação nutricional fornecida a educadores infantis de escola particular no município de São Paulo, encontrou aumento significativo no conhecimento destes após a intervenção, além de depoimentos de pais e alunos revelando mudanças positivas ocorridas no comportamento alimentar destes últimos.

Vale ressaltar que a American Dietetic Association sugere que especialistas prestem assistência às escolas no desenvolvimento de programas educativos em nutrição, a fim de apoiar padrões de qualidade e, certamente, produzir melhores resultados ${ }^{9}$. Reforçando essa ideia, Gaglianone $^{8}$ cita que para o aumento da efetividade da educação nutricional no ambiente escolar é necessária uma ação integrada dos profissionais de saúde e educação no planejamento das atividades, evitando que ações educativas para a promoção da saúde se tornem uma exigência adicional e uma sobrecarga à prática profissional do educador.

Os resultados encontrados neste estudo corroboram tais citações, indicando que a assistência de especialista, caracterizando uma ação integrada, maximizou os resultados do programa "Criança Saudável, Educação Dez". Entretanto, há que se admitir que, considerando que o programa de orientação aos professores foi desenvolvido pela mesma pesquisadora que elaborou o instrumento de avaliação do conhecimento dos escolares, um viés de indução pode ter ocorrido. Porém, tal preocupação pode ser minimizada com base no fato de que o conteúdo do projeto educativo foi desenvolvido pelos professores, cada qual com sua especificidade pedagógica, diluindo assim esse possível viés.

Powers et al. ${ }^{16}$ observaram que, comparados ao grupo-controle, os escolares de nível fundamental expostos a programa de educação nutricional exibiram aumento significante no conhecimento sobre nutrição, incluindo compreensão da pirâmide alimentar, capacidade de associar nutriente e alimento, bem como conhecimento das funções dos nutrientes. Estudando conhe- cimentos, práticas e atitudes de escolares em relação à alimentação, Foley et al. ${ }^{17}$ observaram que escolares com melhor conhecimento em nutrição eram os que tinham atitudes e práticas alimentares mais positivas. Entretanto, não se tem claro o quanto o conhecimento interfere nos hábitos alimentares. Porém, acredita-se que conhecimento e competência sejam necessários para o estabelecimento de comportamentos desejados. Sabe-se que hábitos alimentares são difíceis de serem mudados, pois, além de envolverem mudanças em antigos padrões, são considerados componentes da história individual, da família e do grupo social ${ }^{18}$.

Nesse sentido, Chapman et al. ${ }^{19}$ citam que o conhecimento sobre o que comer é um primeiro degrau na influência do comportamento alimentar saudável; ele não instiga a mudança, mas funciona como um instrumento quando as pessoas desejam mudar, o que, para Baldwin \& Falciglia ${ }^{20}$, exige ainda a modificação de fatores pessoais internos que incluem o desenvolvimento de autoeficácia, autocontrole e autoavaliação.

A associação entre obesidade, práticas alimentares e conhecimentos de nutrição foi avaliada por Triches \& Giuliani ${ }^{21}$ em 573 escolares matriculados em escolas municipais de um município do Rio Grande do Sul. Os autores observaram que a obesidade mostra-se associada com menor nível de conhecimento de nutrição e práticas alimentares menos saudáveis, acrescentando que crianças com essas características apresentam cinco vezes mais chances de serem obesas.

Considerando a importância da educação nutricional e seu papel no contexto das Diretrizes para a Promoção da Alimentação Saudável nas Escolas $^{5}$, considerando a necessidade de medidas contra o avanço das Doenças Crônicas Não-Transmissíveis e considerando ainda que melhores resultados são alcançados com a participação de nutricionistas, cabe discutir a atenção dada ao componente educação na formação desses profissionais.

As Diretrizes Curriculares do Curso de Graduação em Nutrição"11, instituídas em 2001, deter- 
minam que os conteúdos curriculares do curso contemplem as ciências sociais e humanas, como áreas que apoiam as abordagens em educação. Entretanto, avaliando 12 cursos de graduação em Nutrição, em 2005, Canesqui \& Garcia12 observaram que as disciplinas Educação Nutricional e Nutrição em Saúde Pública, quando unidas numa mesma categoria, ocupavam em média apenas 4,7\% da carga horária total dos cursos.

Ademais, percebe-se a tendência dos currículos a atribuir a responsabilidade pela formação em educação apenas à disciplina Educação Nutricional e a seus docentes. Nesse sentido, Achterberg ${ }^{22}$ cita que em educação nutricional há necessidade de a pesquisa, a teoria e a prática estarem combinadas a fim de facilitar o ensino. De outro lado, porém, Franco \& Boog ${ }^{23}$ observaram que, entre os professores de Educação Nutricional entrevistados, não há consenso sobre o significado atribuído à atividade prática da disciplina nem de como ela deve ser realizada.

Diante dos resultados deste estudo e da discussão apresentada, pode-se crer que o campo pedagógico merece maior aprofundamento nos cursos de formação de nutricionistas, devendo ser abordado num conjunto de disciplinas que possam apoiar uma compreensão alicerçada e sólida do processo educativo, e não somente numa única disciplina. Tais reflexões visam ao aprimoramento do ensino e, por conseguinte, da formação dos futuros profissionais, com vista a uma participação mais efetiva da categoria no campo político e social.

\section{O N CLUS Ã O}

Programas de educação nutricional desenvolvidos no ambiente escolar apresentaram melhores resultados sobre o conhecimento de escolares da $1^{\text {a }}$ à $4^{a}$ série do ensino fundamental quando algumas condições foram estabelecidas, como a realização de um programa de orientação aos professores, possibilitando discussões semanais como forma de preparação e planejamento das atividades, o que também gerou maior comprometimento da direção escolar e da equipe pedagógica.

A realização do programa de orientação aos professores para o desenvolvimento do projeto educativo, realizado por nutricionista coordenando e apoiando a abordagem dos assuntos, assim como propondo estratégias educativas, maximizou os resultados, gerando mudança considerada significante no conhecimento dos escolares sobre alimentação e nutrição.

\section{COLABORADORES}

Ambos os autores participaram de todas as fases da redação do artigo.

\section{REFERÊ N CIAS}

1. Brasil. Ministério da Saúde. Política nacional de alimentação e nutrição. $2^{a}$ ed. Brasília: Ministério da Saúde; 2003.

2. World Health Organization. Global strategy on diet, physical activity and health. Fifty-Seventh World Health Assembly - WHA57.17. Geneva: WHO; 2004.

3. Murphy AS, Youatt JP, Hoerr SL, Sawyer CA, Andrews SL. Kindergarten students' food preferences are not consistent with their knowledge of the dietary guidelines. J Am Diet Assoc. 1995; 95(2):219-23.

4. Vítolo MR, Gaglianone CP, Grazini JT. Educação nutricional. In: Nóbrega FJ. Distúrbios da nutrição. Rio de Janeiro: Revinter; 1998. p.42-4.

5. Brasil. Ministério da Saúde e Ministério da Educação. Portaria Interministerial n ${ }^{\circ} 1.010$, de 8 de maio de 2006. Brasília, 2006 [acesso 2008 jun 12]. Disponível em: <http://dtr2004.saude.gov.br/sas/ legislação/portaria1010_08_05_06.pdf>.

6. Brasil. Ministério do Desenvolvimento Social e Combate a Fome Programa criança saudável, educação dez. Brasília; 2005 [acesso 2010 mar 29]. Disponível em: <http://www.mds.gov.br/programas/ seguranca-alimentar-e-nutricional-san/educacaoalimentar-e-nutricional/projetos/crianca_saudavel__educacao_dez_mds_-_editora_globo>.

7. Boog MCF. Educação nutricional: passado, presente e futuro. Rev Nutr. 1997; 10(1):5-19. 
8. Gaglianone CP. Projeto redução dos riscos de adoecer e morrer na maturidade: prevenção da obesidade entre crianças do ensino público fundamental de São Paulo [tese]. São Paulo: Escola Paulista de Medicina; 2003.

9. American Dietetic Association. Position of the American Dietetic Association: local support for nutrition integrity in schools. J Am Diet Assoc. 2006; 106(1):122-33.

10. Bizzo MLG, Leder L. Educação nutricional nos parâmetros curriculares nacionais para o ensino fundamental. Rev Nutr. 2005; 18(5):661-67. doi:10.15 90/S1415-52732005000500009.

11. Brasil. Conselho Nacional de Educação. Câmara de Educação Superior. Resolução n.5 de 7 de novembro de 2001. Institui Diretrizes Curriculares Nacionais do Curso de Graduação em Nutrição. Brasília; 2001.

12. Canesqui AM, Garcia RWD. Ciências sociais e humanas nos cursos de nutrição. In: Canesqui AM, Garcia RWD, organizadores. Antropologia e nutrição: um diálogo possível. Rio de Janeiro: Fiocruz; 2005. p.255-74.

13. Vieira S. Introdução à bioestatística. $3^{\mathrm{a}} \mathrm{ed}$. Rio de Janeiro: Elsevier; 1980.

14. Davanço GM, Taddei JAAC, Gaglianone CP. Conhecimentos, atitudes e práticas de professores de ciclo básico, expostos e não expostos a Curso de Educação Nutricional. Rev Nutr. 2004; 17(2): 177-84. doi: 10159/S1415-52732004000200004.

15. Macedo IC. Capacitação para educação nutricional dirigida a professores de um curso de educação infantil [mestrado]. São Paulo: Universidade de São Paulo; 2003.
16. Powers AR, Struempler BJ, Guarino A, Farmer SM. Effects of a nutrition education program on the dietary behavior and nutrition knowledge of second-grade and third-grade students. J Sch Health. 2005; 75(4):129-33.

17. Foley CS, Vaden AG, Newell GK, Dayton AD. Establishing the need for nutrition education: III. Elementary students' nutrition knowledge, attitudes, and practices. J Am Diet Assoc. 1983; 83(5):564-8.

18. Freitas MCS. Educação nutricional: aspectos sócio-culturais. Rev Nutr. 1997; 10(1):45-9.

19. Champman KM, Ham JO, Liesen P, Winter L. Applying behavioral models to dietary education of eldery diabetic patients. J Nutr Educ. 1995; 27(2): 75-9.

20. Baldwin TT, Falciglia GA. Application of cognitive behavioral theories to dietary change in clients. J Am Diet Assoc. 1995; 95(11):1315-17.

21. Triches RM, Giugliani ERJ. Obesidade, práticas alimentares e conhecimentos de nutrição em escolares. Rev Saúde Pública. 2005; 39(4):541-47.

22. Achterberg C. A perspective on nutrition education research and practice. J Nutr Educ. 1988; 20(5): 240-3.

23. Franco AC, Boog, MCF. Relação teoria-prática no ensino de educação nutricional. Rev Nutr. 2007; 20(6):643-55. doi:10.1590/\$1415-527320070006 00007.
Recebido em: 12/5/2008

Versão final reapresentada em: 4/5/2010 Aprovado em: 14/6/2010 
